\title{
Prone-only SPECT myocardial perfusion imaging: An alternative standard in clinical practice?
}

\author{
Valeria Cantoni, PhD, ${ }^{\mathrm{a}}$ Roberta Green, $\mathrm{PhD},{ }^{\mathrm{a}}$ and Alberto Cuocolo, $\mathrm{MD}^{\mathrm{a}}$ \\ a Department of Advanced Biomedical Sciences, University Federico II, Naples, Italy
}

Received Oct 15, 2020; accepted Oct 15, 2020

doi: $10.1007 / \mathrm{s} 12350-020-02423-7$

\section{See related article, pp. 1339-1351}

Myocardial perfusion imaging (MPI) using single photon emission computed tomography (SPECT) is the most common non-invasive test for diagnosis of coronary artery disease (CAD), risk stratifying of patients after infarction, assessing myocardial viability, and planning therapy. ${ }^{1}$ Coronary angiography is still the gold standard test for detecting CAD; however, this is an invasive method with a broad range of risks and complications (such as hemorrhage, endothelial injuries, and hematoma). ${ }^{2}$ SPECT-MPI is usually performed with the patient in the supine position with the arms raised above the head and supported. ${ }^{3,4}$ It is, however, recognized that the diaphragmatic attenuation of the inferior wall and the breast attenuation of the anterior wall in females has an impact on the test specificity. ${ }^{1,3-7}$ In addition, there is potential for loss of sensitivity, as perfusion defects may be interpreted as attenuation artifacts. Planar acquisition, prone imaging, ECG gating, and image quantitation constitute commonly used approaches to overcome soft tissue attenuation. Although direct approaches for attenuation correction have been commercially available, they are quite expensive and possibly not provided to all nuclear medicine departments. ${ }^{1,8}$ Prone imaging has been shown to reduce patient motion and reduce inferior wall attenuation, improve the specificity and accuracy of inferior wall defect detection, and decrease the frequency and degree of patient motion. ${ }^{9-11}$ Segall

Reprint requests: Alberto Cuocolo, MD, Department of Advanced Biomedical Sciences, University Federico II, Via Pansini 5, 80131 Naples, Italy; cuocolo@unina.it

J Nucl Cardiol 2022;29:1352-5.

1071-3581/\$34.00

Copyright (C) 2020 American Society of Nuclear Cardiology. et al. ${ }^{9,10}$ ) initially described SPECT-MPI with data acquisition in the prone position. Subsequently, other studies with ${ }^{201} \mathrm{Tl}$ or ${ }^{99 \mathrm{~m}} \mathrm{Tc}$-sestamibi SPECT-MPI showed that imaging acquisition in the prone position improves the specificity for evaluating inferior wall abnormalities by minimizing diaphragmatic attenuation. ${ }^{12,13}$ However, the routine change of supine to prone imaging is a controversial matter, given the occasionally seen artifactual anterior-anteroseptal wall prone defect. ${ }^{11,13,14}$ This finding is presumably attributed to sternal and/or rib attenuation. ${ }^{1,4}$ The majority feels that prone should be considered only when imaging in the supine position raises the question of true inferior wall perfusion defect or artifact abnormality.,11,13 No evidence from larger series is available as to which position is better in routine work. If interpretation of a study obtained in the supine position is hampered by infradiaphragmatic attenuation or scatter, an additional acquisition in the prone position may be tried. As Taasan and colleagues ${ }^{15}$ correctly point out the benefits of prone imaging include downward displacement of the diaphragm and abdominal organs, compression of anterior chest soft tissue including breast tissue, a shift of the heart more anteriorly, and reduction of patient motion. There is no doubt that attenuation correction decreases equivocal studies compared to prone imaging. ${ }^{16}$ However, in the same study utilizing ${ }^{99 \mathrm{~m}}$ Tc- based rest and stress imaging with attenuation correction, prone imaging, and prone and supine imaging without attenuation correction, prone imaging significantly reduced equivocal studies. ${ }^{16}$ Indeed, even with a state-of-the-art SPECT-CT system imaging may reduce the incidence of apical artifacts. ${ }^{17}$ Stathaki et al. ${ }^{18}$ showed that the addition of prone position to stress supine myocardial scintigraphy decreases the false-positive rates and leads to more accurate results. The prone position, which moves the diaphragm downward and the heart upward, enlarges the distance between the inferior wall of the left ventricle and diaphragm, and minimizes diaphragmatic 
attenuation. $^{19}$ Furthermore, it increases specificity without compromising sensitivity for the diagnosis of CAD. ${ }^{18}$ Segall and Davis ${ }^{10}$ demonstrated that specificity for right coronary artery was dramatically better $(90 \%$ versus 66\%) when patients were submitted to prone image acquisition compared to supine. Moreover, the overall effect on the detection of CAD was an improved accuracy and higher specificity (82\% versus 59\%) without significant loss of sensitivity (75\% versus $79 \%)$. In addition, Hayes et al. ${ }^{4}$ concluded that patients with inferior wall defect in the supine position that was not present in the prone image had similar low risk of cardiac events, when compared with those that had normal supine only studies. Katayama et al. ${ }^{20}$ demonstrated the diagnostic accuracy of ${ }^{201} \mathrm{Tl}$ stress prone SPECT images for detecting CAD in the inferior wall of the left ventricle compared with that of stress-rest supine images. In particular, they found a tendency toward improved specificity and decreased false-positive rates. Shin et al. ${ }^{14}$ in a large study examined the ability CAD as determined by coronary angiography. They found that prone SPECT-MPI demonstrated high sensitivity for detection of CAD (92\% for stenosis $\geq 70 \%)$ and high normalcy rate $(95 \%)$. They also found no significant difference in the normalcy rate among the three coronary distributions when assessed in the prone position. ${ }^{14}$ As is standard practice in nuclear cardiology reports, normalcy rate was used as a proxy for specificity given the inherent post-test referral bias in populations of patients who have been selected after referral for cardiac catheterization after an abnormal SPECT-MPI. Their results suggest that a prone-only imaging protocol can be considered a reasonable option for SPECT-MPI. It seems that prone imaging might have an additional role in preventing unnecessary coronary angiograms and minimize radiation exposure, especially in low-risk patients. Recently, Worden et al. ${ }^{21}$ showed that patients with perfusion abnormalities during stress supine imaging that resolved during prone imaging are at low risk for cardiac death or myocardial infarction at medium-term follow up. Given that they seldom require invasive coronary angiography, broader application of prone imaging could lead to reduced exposure to the risks and expenses of unnecessary invasive procedures. $^{21}$ Anterior wall defects are most common in women. Although some believe that positional change mainly contributes to the disappearance rate of diaphragmatic attenuation, ${ }^{11,22}$ it is a confirmed knowledge that combined supine and prone approach improves specificity and normalcy rates in women. Slomka et al. ${ }^{23}$ reported no differences in normalcy rates between prone and supine acquisitions in a population of women. In this study, they also find similar normalcy rates in each of the three coronary distributions, further suggesting that these concerns may be overstated. More recently, the new cadmium zinc telluride (CZT) cameras have made a significant difference in evaluation of patients for CAD. ${ }^{24,25}$ Nishiyama et al. ${ }^{26}$ assessed the feasibility of combined imaging using a novel ultrafast CZT camera. They concluded that the combined supine and prone CZT SPECT yields significant gains in specificity and accuracy, whereas acquisition time is reduced by up to one fifth. However, troublesome artifact effects of the inferior wall exist similarly to those on images obtained with conventional Anger cameras.

In the current issue of the Journal, Mirshahvalad et al. ${ }^{27}$ conducted a systematic review and meta-analysis aimed to evaluate the diagnostic performance of the prone position in detection of CAD as an independent standard. The authors reviewed and analyzed 10 articles evaluated the prone position MPI as a diagnostic method to detect the CAD. They included studies that defined CAD with coronary angiography, using the threshold of $\geq 50 \%$ stenosis and provided adequate data to extract the sensitivity, specificity, positive predictive value, negative predictive value, and accuracy of SPECT-MPI in detecting CAD. They separated the articles that provided data for the prone position in all territories (PAT) and the right coronary artery territory (PRT). Their results showed that the pooled sensitivities of the studies were $83 \%$ (95\% confidence interval, CI 0.79-0.86), 70\% (95\% CI $0.59-0.79$ ), and $86 \%$ (95\% CI 0.83-0.88) for PAT, PRT, and supine all territories (SAT), respectively. The pooled specificities of the studies were $79 \%(95 \% \mathrm{CI}$ $0.72-0.85), 84 \%$ (95\% CI 0.79-0.88), and 67\% (95\% CI 0.59-0.74) for PAT, PRT, and SAT, in turn. ${ }^{27}$ The results of this meta-analysis emphasize the role of prone-only SPECT-MPI. The authors showed that in the suspicion for the $\mathrm{CAD}$, prone position with comparable sensitivity and higher specificity can be an acceptable alternative to the supine position as the standard method, also in the cases of possible defects in the right coronary artery territory. ${ }^{27}$ Despite the benefits of prone imaging, the specific manner in which it should be employed in standard clinical practice remains unclear. Many laboratories currently perform prone-only imaging for patients who are unable to lie supine or when severe diaphragmatic attenuation is expected. Recent guidelines of the American Society of Nuclear Cardi$\operatorname{ology}^{28}$ ) recommend supine position as the standard for SPECT- MPI with most currently available Anger and CZT systems; the routine use of 2-position imaging, combination of supine followed by prone acquisition or upright and supine imaging, is recommended, at least for stress images, particularly if attenuation correction is not available. By comparing supine and prone images, artefactual defects will resolve or change their location whereas true perfusion defects will remain in the same 
position. ${ }^{3,13}$ When being used in this fashion, the acquisition time for the secondary (prone) image set can be reduced by $20 \%$ to $40 \%{ }^{26}$ Moreover, the addition of prone position to stress supine myocardial scintigraphy results in the key benefit of reducing the number of unnecessary rest studies performed, while minimizing radiation exposure, investigation time, and costs. ${ }^{18}$ It could possibly be a useful and practical method of obviating unnecessary referrals to coronary angiograms, especially in low-risk patients. ${ }^{18}$ In this scenario, prone SPECT-MPI should not be used alone, making the routine change of supine to prone imaging still debated. Therefore, further studies in larger patient populations are needed to standardize the use of prone SPECT-MPI in clinical routine practice.

\section{Disclosure}

Valeria Cantoni, Roberta Green, and Alberto Cuocolo declare that they have no conflicts of interest.

\section{References}

1. Bateman TM, Cullom SJ. Attenuation correction single-photon emission computed tomography myocardial perfusion imaging. Semin Nucl Med 2005;35:37-51.

2. Tavakol M, Ashraf S, Brener SJ. Risks and complications of coronary angiography: a comprehensive review. Glob J Health Sci 2012;4:65-93.

3. Nishina H, Slomka PJ, Abidov A, Yoda S, Akincioglu C, Kang X, et al. Combined supine and prone quantitative myocardial perfusion SPECT: method development and clinical validation in patients with no known coronary artery disease. J Nucl Med 2006; $47: 51-8$.

4. Hayes SW, De Lorenzo A, Hachamovitch R, Dhar SC, Hsu P, Cohen I, et al. Prognostic implications of combined prone and supine acquisitions in patients with equivocal or abnormal supine myocardial perfusion SPECT. J Nucl Med 2003;44:1633-40.

5. Stowers SA, Umfrid R. Supine-prone SPECT myocardial perfusion imaging: the poor man's attenuation compensation. J Nucl Cardiol 2003;10:338.

6. Cuocolo A. Attenuation correction for myocardial perfusion SPECT imaging: still a controversial issue. Eur J Nucl Med Mol Imaging 2011;38:1887-9.

7. Mannarino T, Assante R, Ricciardi C, Zampella E, Nappi C, Gaudieri V, et al. Head-to-head comparison of diagnostic accuracy of stress-only myocardial perfusion imaging with conventional and cadmium-zinc telluride single-photon emission computed tomography in women with suspected coronary artery disease. J Nucl Cardiol 2019. https://doi.org/10.1007/s12350-019-01789-7.

8. Hedén B, Persson E, Carlsson M, Pahlm O, Arheden H. Disappearance of myocardial perfusion defects on prone SPECT imaging: comparison with cardiac magnetic resonance imaging in patients without established coronary artery disease. BMC Med Imaging 2009;9:16.

9. Segall GM, Davis MJ, Goris ML. Improved specificity of prone versus supine thallium SPECT imaging. Clin Nucl Med 1988;13:915-6.
10. Segall GM, Davis MJ. Prone versus supine thallium myocardial SPECT: A method to decrease artifactual inferior wall defects. J Nucl Med 1989;30:548-55.

11. Kiat H, Van Train KF, Friedman JD, Germano G, Silagan G, Wang FP, et al. Quantitative stress-redistribution thallium-201 SPECT using prone imaging: Methodologic development and validation. J Nucl Med 1992;33:1509-15.

12. Schoss RM, Gorten RJ. Comparison of supine versus prone tomographic myocardial imaging: effect on false-positive rate. Clin Nucl Med 1996;21:445-51.

13. Berman D, Germano G, Lewin H, Kang X, Kavanagh PB, Tapnio $\mathrm{P}$, et al. Comparison of post-stress ejection fraction and relative left ventricular volumes by automatic analysis of gated myocardial perfusion single-photon emission computed tomography acquired in the supine and prone positions. J Nucl Cardiol 1998;5:40-7.

14. Shin JH, Pokharna HK, Williams KA, Mehta R, Ward RP. SPECT myocardial perfusion imaging with prone-only acquisitions: correlation with coronary angiography. J Nucl Cardiol 2009;16:590-6.

15. Taasan V, Wokhlu A, Taasan MV, Dusaj RS, Mehta A, Kraft S, et al. Comparative accuracy of supine-only and combined supineprone myocardial perfusion imaging in men. J Nucl Cardiol 2016;23:1470-6.

16. Malkemeker D, Brenner R, Martin WH, et al. CT-based attenuation correction versus prone imaging to decrease equivocal interpretation of rest/stress Tc-99m tetrofosmin SPECT MPI. J Nucl Cardiol 2007;14:314-23.

17. Takamura T, Horiguchi Y, Kanna M, Matsushita H, Sudo Y, Kikuchi S, et al. Validation of prone myocardial perfusion SPECT with a variable-focus collimator versus supine myocardial perfusion SPECT with or without computed tomography-derived attenuation correction. Ann Nucl Med 2015;29:890-6.

18. Stathaki M, Koukouraki S, Papadaki E, Tsaroucha A, Karkavitsas $\mathrm{N}$. The benefits of prone SPECT myocardial perfusion imaging in reducing both artifact defects and patient radiation exposure. Arq Bras Cardiol 2015;105:345-52.

19. DePuey EG. How to detect and avoid myocardial perfusion SPECT artifacts. J Nucl Med 1994;35:699-702.

20. Katayama T, Ogata N, Tsuruya Y. Diagnostic accuracy of supine and prone thallium-201 stress myocardial perfusion single-photon emission computed tomography to detect coronary artery disease in inferior wall of left ventricle. Ann Nucl Med 2008;22:317-21.

21. Worden NE, Lindower PD, Burns TL, Chatterjee K, Weiss RM. A second look with prone SPECT myocardial perfusion imaging reduces the need for angiography in patients at low risk for cardiac death or MI. J Nucl Cardiol 2015;22:115-22.

22. Perault C, Loboguerrero A, Liehn JC, Wampach H, Gibold C, Ouzan J, et al. Quantitative comparison of prone and supine myocardial SPECT MIBI images. Clin Nucl Med 1995;20:678-84.

23. Slomka PJ, Nishina H, Abidov A, Hayes SW, Friedman JD, Berman DS, et al. Combined quantitative supine-prone myocardial perfusion SPECT improves detection of coronary artery disease and normalcy rates in women. J Nucl Cardiol 2007;14:44-52.

24. Cantoni V, Green R, Acampa W, Zampella E, Assante R, Nappi C, et al. Diagnostic performance of myocardial perfusion imaging with conventional and CZT single-photon emission computed tomography in detecting coronary artery disease: A meta-analysis. J Nucl Cardiol 2019. https://doi.org/10.1007/s12350-019-01747-3.

25. Cantoni V, Green R, Ricciardi C, Assante R, Zampella E, Nappi C, et al. A machine learning-based approach to directly compare the diagnostic accuracy of myocardial perfusion imaging by conventional and cadmium-zinc telluride SPECT. J Nucl Cardiol 2020. h ttps://doi.org/10.1007/s12350-020-02187-0.

26. Nishiyama Y, Miyagawa M, Kawaguchi N, Nakamura M, Kido T, Kurata A, et al. Combined supine and prone myocardial perfusion 
single-photon emission computed tomography with a cadmium zinc telluride camera for detection of coronary artery disease. Circ J 2014;78:1169-75.

27. Mirshahvalad SA, Chavoshi M, Hekmat S. Diagnostic performance of prone-only myocardial perfusion imaging versus coronary angiography in the detection of coronary artery disease: A systematic review and meta-analysis. J Nucl Cardiol 2020. h ttps://doi.org/10.1007/s12350-020-02376-X.
28. Dorbala S, Ananthasubramaniam K, Armstrong IS, Chareonthaitawee P, DePuey EG, Einstein AJ, et al. Single photon emission computed tomography (SPECT) myocardial perfusion imaging guidelines: Instrumentation, acquisition, processing, and interpretation. J Nucl Cardiol 2018;25:1784-846.

Publisher's Note Springer Nature remains neutral with regard to jurisdictional claims in published maps and institutional affiliations. 